\title{
Detection of $\beta$-lactamase Mediated Resistance among Pseudomonas aeruginosa Isolates from Ocular Infections
}

\section{ABSTRACT}

Introduction: P.aeruginosa is the most prevalent gramnegative organism in ocular infections. Production of extended spectrum of $\beta$-lactamases (ESBL) by them may require alteration in the management protocol of gram-negative ocular infections.

Aim: To analyze the prevalence and detection of $\beta$-lactamase producing $P$.aeruginosa from ocular infections.

Materials and Methods: This study included all the patients with ocular infections who visited a tertiary care eye hospital in Coimbatore, for a period of four months. During this study period, the isolates were confirmed as P.aeruginosa by standard identification protocol. Minimum inhibitory concentration (MIC) of the antibiotics was determined by agar dilution method. Inducible $\beta$-lactamases and extended spectrum $\beta$-lactamases were detected by double disc diffusion method.
Results: Of the 2644 ocular specimens collected from 2618 patients, a total of 34 isolates of P.aeruginosa were obtained. MICs of cefotaxime and ceftazidime for all the isolates revealed that, $21(62 \%)$ isolates were resistant to cefotaxime. A total of 11 strains were induced to produce $\beta$-lactamase with disc approximation at $20 \mathrm{~mm}$ apart and the presence of ESBL was detected in $4.3 \%$ of strains through $20 \mathrm{~mm}$ disc approximation, $34.7 \%$ of strains through $15 \mathrm{~mm}$ disc approximation. The synergic effect of cefotaxime with aminoglycosides was seen among $61.7 \%$ of strains.

Conclusion: Resistance of $P$.aeruginosa to $\beta$-lactam antibiotics seems to be prevalent in this part of the state. Combination of cefotaxime with aminoglycosides may be more useful for treatment of severe eye infections caused by $P$. aeruginosa.

\section{INTRODUCTION}

Pseudomonas aeruginosa is a significant pathogen among gram-negative organisms infecting eye [1,2], because of its wide prevalence and resistance to antibiotics. In recent years, its resistance to $\beta$-lactam antibiotics and extended spectrum cephalosporins is of great concern to ophthalmologists worldwide. Pseudomonas resists $\beta$-lactam antibiotics by synthesizing $\beta$-lactamases [3]. These enzymes inactivate cephalosporins by hydrolyzing the amide bond of the $\beta$-lactam ring. The subsequent generation cephalosporins, which could overcome $\beta$-lactamases, are called extended spectrum cephalosporins, which include oxyimino $\beta$-lactams like ceftazidime and cefotaxime. Resistance to these antibiotics is by synthesis of extended spectrum $\beta$-lactamases (ESBLs), which are plasmid-mediated enzymes. These enzymes differ from their parent enzymes by only a few amino acid position but can hydrolyze extended spectrum cephalosporins [3].

Though these plasmid-mediated $\beta$-lactamases are observed in $P$. aeruginosa isolates in less than $2 \%$, new classes of chromosomally encoded extended spectrum $\beta$-lactamases are also reported [3]. These enzymes have moderate hydrolysis activity for oxacillin but high activity against extended spectrum cephalosporins i.e., cefotaxime and ceftazidime.
Keywords: Antibiotics, Eye infections, Susceptibility

The present study was therefore carried out in a tertiary care eye hospital in Coimbatore, south India to isolate $P$. aeruginosa from patients with ocular infections and to detect the synthesis of ESBL and their antimicrobial susceptibility pattern.

\section{MATERIALS AND METHODS}

The study was designed as a retrospective, laboratory based investigation which involved a total of 2644 (including repeat specimens) specimens (viz., corneal swab and corneal scraping) from 2618 patients processed from the patients attending Aravind Eye Hospital and Postgraduate Institute of Ophthalmology Coimbatore, India during January to April 2004. All the specimens that showed positive for bacteria in direct microscopy and culture media were included and specimens that grew fungal colonies were excluded from the study. The specimens were directly streaked onto solid media such as blood agar, chocolate agar, potato dextrose agar (PDA) and brain heart infusion broth and the plates/tubes were incubated at $37^{\circ} \mathrm{C}$ for 24 hours. The specimens were also placed onto clean, scratch free and labeled slide for gram staining. After incubation, the colonies were further subjected for gram staining and standard biochemical tests $[4,5]$ so as to identify the causative organisms. 
The isolates were stored as suspensions in a $10 \%$ (wt/vol) skimmed milk solution containing 10\% (vol/vol) glycerol at $-20^{\circ} \mathrm{C}$ until additional tests were performed. Only the confirmed $P$. aeruginosa strains isolated from ocular infections available from the storage facility $\left(-20^{\circ} \mathrm{C}\right)$ were used in the study and none of the patient was directly involved.

\section{Determination of minimuminhibitory concentration (MIC)}

MIC of the antibiotics, cefotaxime and ceftazidime were determined by agar dilution technique [6,7]. Muller-Hinton agar containing cefotaxime and ceftazidime in final concentrations of $512,256,128,64,32,16,8,4,2,1,0.5,0.25$ and $0 \mu \mathrm{g} / \mathrm{ml}$ were used for the analysis. The MIC was defined as the lowest concentration of the drug inhibiting visible bacterial growth as evaluated after 18 hours incubation and the MIC breakpoints were interpreted according to the Clinical and Laboratory Standards Institute (CLSI) guidelines [6]. P.aeruginosa ATCC 27853 was used as a reference strain in every batch of MIC tests.

\section{Detection of group I inducible $\beta$-lactamases}

The prevalence of inducible $\beta$-lactamases was investigated by disc approximation method $[7,8]$. Cefotaxime $(30 \mu \mathrm{g})$ disc was placed at distances $25 \mathrm{~mm}$ and $20 \mathrm{~mm}$, respectively from a central cefoxitin $(30 \mu \mathrm{g})$ disc on Muller-Hinton agar (MHA) plate swabbed with the lawn culture of the test isolate. After overnight incubation, distinct flattening of the inhibitory zone around the ceftazidime disc on the side nearest to the cefoxitin disc was regarded as an indication for the presence of inducible $\beta$-lactamase.

\section{Detection of extended spectrum $\beta$-lactamase (ESBL) activity}

The isolates were screened for the presence of ESBLs by the

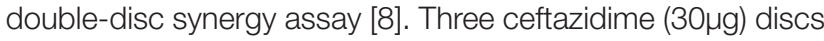
were placed at distances 20,15, and $10 \mathrm{~mm}$, respectively, from a central amoxicillin-clavulanic acid disc on MHA swabbed with the lawn culture of the test isolate and the plates were incubated at $37^{\circ} \mathrm{C}$ overnight. The test result was considered positive when an enhancement of the inhibition zone around at least one of the ceftazidime disc toward the clavulanic-acid disc was observed [9].

\section{Investigation of synergy effects of antibiotic combinations}

The synergy effects of the antibiotic combinations against the selected isolates were examined by disc diffusion test $[8,10]$. Two discs, each containing one or other of the two tested antibiotics (clavulanic acid and cefotaxime), were placed at a distance of about $20 \mathrm{~mm}$ from each other on MHA agar plate swabbed with the lawn culture of the test isolate. Synergy was considered to occur when there was a well-observed change $(<2 \mathrm{~mm})$ in the zone of inhibition. The synergy was classified as weak when a change $<2 \mathrm{~mm}$ was observed in the zone of inhibition [10].

\section{RESULTS}

Most of the specimens 2079 (79\%) were preoperative screening conjunctival swabs and only 108 (5\%) specimens showed bacterial growth. Of these, $P$. aeruginosa grew in 3 (2.7\%) cultures [Table/Fig-1a]. Only 565 (21\%) specimens from 539 patients were processed for ocular infections.

P.aeruginosa grew in 34 cultures. Of these 21 (61.7\%) isolates were cultured from corneal ulcer scrapings. MIC analysis revealed that, $21(62 \%)$ isolates were found to be resistant to cefotaxime and 5 (15\%) strains were found to be resistant to ceftazidime.

Isolates showing $\mathrm{MIC}$ value $\geq 16 \mathrm{mg} / \mathrm{L}$ were considered resistant for cefotaxime and ceftazidime (includes intermediate and resistant categories) [6]. In the present study 21 (61.7\%) isolates showed resistance to cefotaxime. Out of these 5 (23.8\%) isolates showed MIC of $\geq 64 \mathrm{mg} / \mathrm{L}$ and $16(76.1 \%)$ isolates had $\mathrm{MIC}$ ranges between $16-32 \mathrm{mg} / \mathrm{L}$ of cefotaxime. When MIC for ceftazidime was tested, a total of $5(14.7 \%)$ isolates were completely resistant, 4 (88\%) and 1 (20\%) isolates had $\mathrm{MIC} \geq 32 \mathrm{mg} / \mathrm{L}$ and $\geq 16 \mathrm{mg} / \mathrm{L}$, respectively.

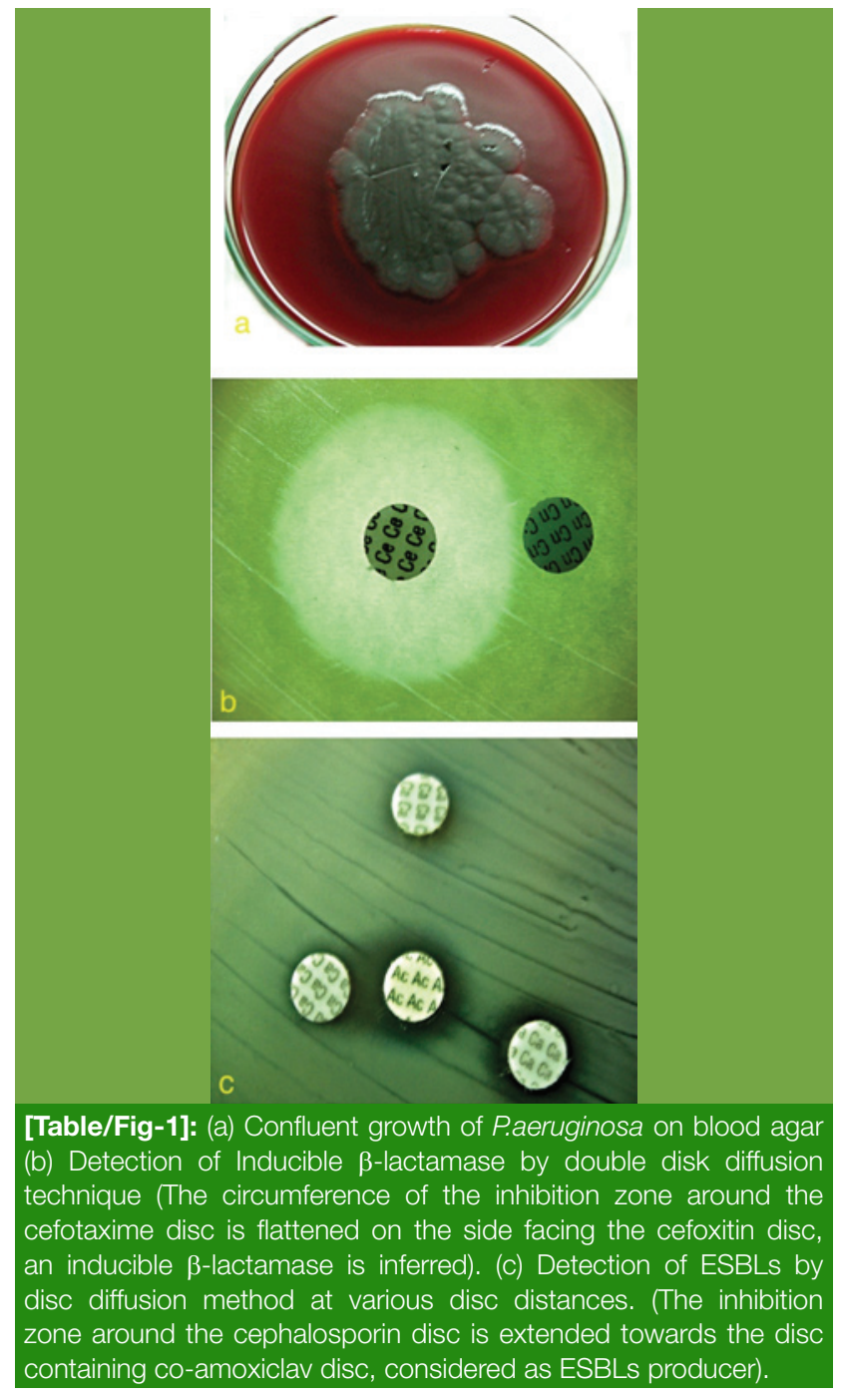


Production of inducible $\beta$-lactamases was detected [Table/ Fig-1b] among 11(32.3\%) isolates, which were sensitive to cephalosporins with a zone of inhibition above $22 \mathrm{~mm}$ diameter. Extended spectrum $\beta$-lactamases were detected [Table/Fig-1c] in $23(67.6 \%)$ isolates, which were resistant as per zone of inhibition. When the two discs (clavulanic acid and cefotaxime) were $25 \mathrm{~mm}$ apart, presence of ESBLs was detected in one (4.3\%) isolate. When the distance was reduced to $15 \mathrm{~mm}, 8(34.7 \%)$ isolates were found to produce ESBLs. At $10 \mathrm{~mm}$ disc approximation, 18 (78.2\%) isolates were found

\begin{tabular}{|c|c|c|}
\hline $\begin{array}{c}\text { Various disc } \\
\text { distances between } \\
\text { Clavulanic acid and } \\
\text { Cephotaxime }\end{array}$ & $\begin{array}{c}\text { No. of positive } \\
\text { isolates (\%) }\end{array}$ & $\begin{array}{c}\text { No. of negative } \\
\text { isolates (\%) }\end{array}$ \\
\hline $25 \mathrm{~mm}$ & $1(4.3)$ & $22(95.6)$ \\
\hline $20 \mathrm{~mm}$ & $1(4.3)$ & $22(95.6)$ \\
\hline $15 \mathrm{~mm}$ & $8(34.7)$ & $15(65.2)$ \\
\hline $10 \mathrm{~mm}$ & $18(78.2)$ & $5(21.7)$ \\
\hline
\end{tabular}

[Table/Fig-2]: Detection of extended spectrum $\beta$-lactamases by double-discsynergy method (resistant isolates $n=23$ ).

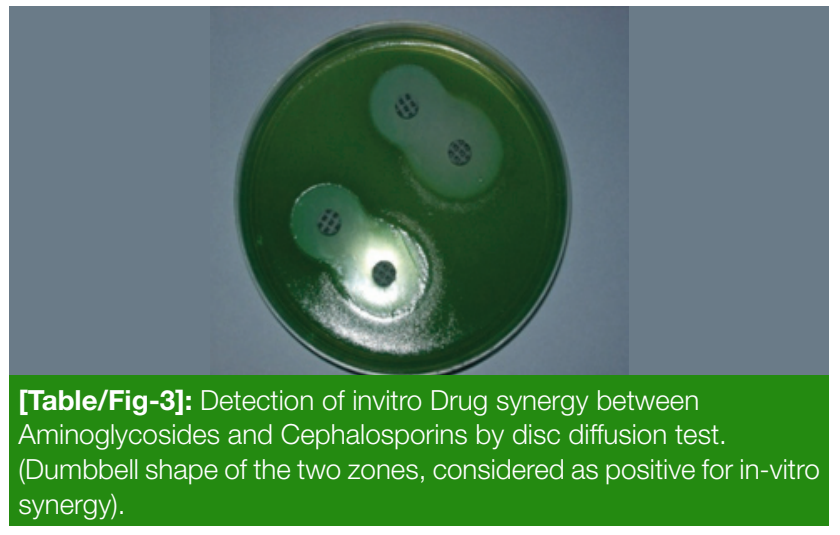

\begin{tabular}{|l|c|c|c|}
\hline \multicolumn{1}{|c|}{ Combination } & $\begin{array}{c}\text { No. } \\
\text { ofisolates } \\
\text { showing } \\
\text { synergy } \\
(\%)\end{array}$ & $\begin{array}{c}\text { No. } \\
\text { ofisolates } \\
\text { showing } \\
\text { weak } \\
\text { synergy } \\
(\mathrm{n} \%)\end{array}$ & $\begin{array}{c}\text { No. } \\
\text { ofisolates } \\
\text { showing } \\
\text { no } \\
\text { synergy } \\
(\mathrm{n} \%)\end{array}$ \\
\hline Amikacin + Cephotaxime & $15(44.1)$ & $6(17.6)$ & $13(38.2)$ \\
\hline Amikacin + Ceftazidime & $13(38.2)$ & $6(17.6)$ & $15(44.1)$ \\
\hline Ofloxacin + Cephotaxime & $17(50)$ & $4(11.7)$ & $13(38.2)$ \\
\hline Ofloxacin + Ceftazidime & $13(38.2)$ & $7(20.5)$ & $14(41.1)$ \\
\hline Ciprofloxacin + Cephotaxime & $17(50)$ & $4(11.7)$ & $13(38.2)$ \\
\hline Ciprofloxacin + Ceftazidime & 13 (38.2) & 7 (20.5) & $14(41.1)$ \\
\hline \\
[Table/Fig-4]: Synergy* effects of combinations of aminoglycosides \\
and fluroquinoloneswith third generation cephalosporins. (Isolates \\
$n=34)$. \\
* Synergy, well-characterized change of $\geq 2$ mm in the inhibition zone. Weak synergy, \\
change of <2 mm in the inhibition zone. No synergy, no change observed in the inhibition \\
zone (Mayer and Nagy, 1999).
\end{tabular}

\begin{tabular}{|c|c|c|}
\hline Demographic Factors & Number of cases & Percentage (\%) \\
\hline \multicolumn{3}{|l|}{ Gender } \\
\hline Male & 18 & 53.0 \\
\hline Female & 16 & 47.0 \\
\hline \multicolumn{3}{|l|}{ Age } \\
\hline $0-20$ & 5 & 14.7 \\
\hline $21-40$ & 11 & 32.3 \\
\hline $41-60$ & 5 & 14.7 \\
\hline $61-80$ & 13 & 38.2 \\
\hline \multicolumn{3}{|l|}{ Occupation } \\
\hline Agricultural & 17 & 50.0 \\
\hline Construction & 7 & 20.5 \\
\hline Students & 3 & 8.8 \\
\hline Office & 2 & 5.8 \\
\hline Unemployed & 5 & 14.7 \\
\hline \multicolumn{3}{|l|}{ Specimen type } \\
\hline Corneal scrapings & 21 & 61.7 \\
\hline Contact lens & 6 & 18 \\
\hline Vitreous & 3 & 9 \\
\hline Conjunctival swab & 3 & 9 \\
\hline Suture infiltrate & 1 & 3 \\
\hline Total & 34 & 100.0 \\
\hline
\end{tabular}

[Table/Fig-5]: Profile of Pseudomonas infected cases taken for a study.

to produce ESBLs. By this method it was found out that 1 (4.3\%) isolate produced only one type of ESBL and the other $18(78.2 \%)$ isolates produced more than one type of ESBLs and 4 (17.3\%) isolates did not produce ESBLs [Table/Fig-2]. Amikacin and ofloxacin when combined with cefotaxime exhibited a synergistic effect [Table/Fig-3] in 21(62\%) on the isolates. When the combinations of amikacin and ofloxacin with ceftazidime were tested by this method, synergy was observed among 20 (58.8\%) isolates [Table/Fig-4]

The epidemiological analysis of the patients revealed the following details. Pseudomonas sp. infections in eye did not show any sex predilection though the males were marginally more affected. Ocular infections by P.aeruginosa were seen among all age groups. The frequency of infection varied in different age group with more cases recorded in 61-80 age group [Table/Fig-5]. Most (50\%) of the patients affected were agricultural workers followed by construction workers (20.5\%).

\section{DISCUSSION}

Ocular infections cause profound morbidity and stand second to cataract in causing blindness worldwide [11]. Though trachoma leads among infective causes of blindness, the other ineffective causes are no less important in ocular infections, because of the morbidity they cause, and also because of 
the increasing antibiotic resistance among bacterial isolates. It is becoming increasingly difficult to control ocular infections through antibiotics. ESBLs are now a problem in hospitalized patients throughout the world.

In this study, it was observed that $14.7 \%$ of strains were resistant to ceftazidime, and $61.7 \%$ of strains were resistant to cefotaxime. Bouza et al., [12] observed a similar finding where, $15 \%$ of the isolates of P.aeruginosa were resistant to ceftazidime. But Joan et al., [13] had reported that 53\% of the Pseudomonas isolates tested were resistant to ceftazidime. Bonomo et al., [14] had reported that $46 \%$ of the Pseudomonas isolates were resistant to cefotaxime, where as in the study conducted by Joan [13], 93\% of the strains were resistant to cephalosporins.

The induction of $\beta$-lactamases was seen in $36 \%$ of the isolates by using standard [7,8] approximation of $20 \mathrm{~mm}$ disc distance. This rate is low when compared to the earlier observation made by Moritz [15] who had demonstrated 68\% of induction for cefotaxime using disc approximation test. But in the present study, all 100\% strains were induced, after decreasing the disc distance between cefoxitin and cefotaxime by $5 \mathrm{~mm}$. This inducibility is significant because, different $\beta$-lactamases are induced at different disc approximations; hence by decreasing the disc distance, we may induce different types of $\beta$-lactamases, thereby proving the capacity of individual strains to induce these enzymes for exhibiting drug resistance [9].

A similar method was followed for detecting the presence of extended spectrum $\beta$-lactamases among resistance strains. By standard disc approximation method $(25 \mathrm{~mm}) \mathrm{ESBL}$ was detected in one isolate, but by reducing disc distances $78.2 \%$ of the isolates were able to produce ESBLs. There are more than 192 types of $\beta$-lactamases discovered [16].These $\beta$-lactamases are produced at different concentration gradients of antibiotics [9]. Some of them are produced even at low level of selective pressure produced by these antibiotics and some require high antibiotic concentration for producing extended $\beta$-lactamases. By altering the disc distances, we can vary the concentrations and alter selective pressures of antibiotics to $P$. aeruginosa. This may be the possible reason for more isolates producing ESBLs at reduced disc approximations. According to Bert et al., [9], the standard test with $30 \mathrm{~mm}$ distance is insufficient to identify most ESBLs produced by Pseudomonas sp. Bradford et al., reported that, TEM derived enzymes and one PER-1 enzyme were detected by use of a $30 \mathrm{~mm}$ distance and it was necessary to reduce the distance to $20 \mathrm{~mm}$ to detect strains that produce VEB-1, SHV 29 and OXA 18. Whereas strains that produce, the other OXA derived ESBLs were identified only at a distance of 10 or $15 \mathrm{~mm}$ [9].

In this study, synergy between cephalosporins and aminoglycosides and cephalosporins and fluroquinolones was detected by disc diffusion method based on Kirby-Bauer's antibiotic susceptibility testing as described by Mayer and Nagy [10]. The synergic effect of cefotaxime with aminglycoside was seen against $61.7 \%$ of strains and the synergic effect of cefotaxime with fluroginolones was also observed against $61.7 \%$ of strains. These combinations may be useful in treating the patients with Paeruginosa but cephalosporin aminoglycoside combination is found to be more nephrotoxic than when drugs are used as mono therapeutic agents [17]. When combination of ceftazidime with aminoglycosides and fluroquinolones were tested against P.aeruginosa, drug synergy was observed in $55.8 \%$ and $58.8 \%$ of isolates, respectively. Though in this study synergy was detected with disc diffusion test, measurement of time killing of the bacteria is the most reliable means of assessing the existence synergic effect between drugs [10]. The main mode of resistance among these organisms is through $\beta$-lactamase production and all strains can be induced to produce $\beta$-lactamases. $P$. aeruginosa can be agreed upon as the most dreaded gramnegative bacteria among ocular isolates and infections are very difficult to treat due to the ability of producing number of virulence factors and antibiotic resistance $[18,19]$.

\section{CONCLUSION}

Our findings suggest that combinations of cefotaxime with aminglycoside may be of significant value for the treatment of severe ocular infections caused by $P$. aeruginosa. Also, the alternative antibiotics such as imipenem may be considered for the unresponsive cases. Moreover, it's mandatory to detect $\beta$-lactamases in all the clinical laboratories, because these enzymes may significantly affect therapeutic outcomes.

\section{REFERENCES}

[1] Aldebasi YH, Aly SM, Ahmad MI, Khan AA. Incidence and risk factors of bacteria causing infectious keratitis. Saudi Med J.2013; 34(11):1156-60.

[2] Singh G, Palanisamy M, Madhavan B, Rajaraman R, Narendran K, Kour A, et al. Multivariate analysis of childhood microbial keratitis in South India. Ann Acad Med Singapore. 2006;35(3):185-89.

[3] Livermore DM. $\beta$-Lactamases in laboratory and clinical resistance. Clin Microbiol Rev. 1995;8(4):557-84.

[4] Forbes BA, Sahm DF, Weissfeld AS: Bailey \& Scott's Diagnostic Microbiology. $11^{\text {th }}$ ed. (Mosby) 2002.

[5] Savitri S. Ocular Microbiology: (Aravind Eye Hospital Publication, Madurai) 1988.

[6] Methods for Dilution Antimicrobial Susceptibility Tests for Bacteria That Grow Aerobically; Approved Standard. The Clinical and Laboratory Standards Institute (CLSI) - Ninth Edition. M07A9. Volume 32 Number 2.

[7] Qin X, Weissman S, Chesnut MF, Zhang B, Shen L. KirbyBauer disc approximation to detect inducible third generation cephalosporin resistance in Enterobacteriaceae. Ann. Clin. Micrbiol. Antimicrob. 2004;3:13.

[8] Collee JG, Fraser AG, Marmion BP, Simmons A. (Eds). Mackie and Mccartney's Practical Medical Microbiology.14th ed. (Churchill Livingstone) 1996.

[9] Bert F, Hocine ZO, Juvin M, Hocin ZO, Juvin M, Dubois V, et al. Evaluation of the Osris Expert system for identification of $\beta$-Lactam phenotypes in isolates of Pseudomonas aeruginosa. J ClinMicrobiol. 2003;41(8):3712-18.

[10] Mayer I, Nagy E. Investigation of the synergic effects of aminoglycoside-fluroquinolone and third-generation cephalosporin 
combinations against clinical isolates of Pseudomonas spp. J Antimicrob Chemother. 1999;43(5):651-57.

[11] Mariotti S, Pascolini D. Visual Impairment, Vision Loss and Blindness 2010 global estimates, and VI and blindness causes. Global Data on Visual Impairments 2010, WHO 2010.

[12] Bouza E, Garrote GF, Cercenado E, Marin M, Diaz MS. Pseudomonas aeruginosa: a survey of resistance in 136 Hospital in Spain. Antimicrob Agents Chemother.1999;43(4):981-82.

[13] Joan FT, Thomas JD, Francis JD, Valerie SJ, Robert EK. Activity of cefepime against ceftazidime and cefotaxime resistant gramnegative bacteria and its relationship to beta lacatmase levels. Antimicrob Agents Chemother.1989;33(4):498-502.

[14] Bonomo RA, Donskey CJ, Blumer JL, Hujer AM, Hoyenm CK, Jacobs MR, et al. Cefotaxime-resistant bacteria colonizing older people admitted to an acute care hospital. J Am Geriatr Soc. 2003;51(4):519-22.
[15] Mortiz VA, Carson PB. Cefoxitin sensitivity as a marker for induicible beta-lactamases. J Med Microbiol. 1986;21(3):20307.

[16] Bradford PA. Extended-Spectrum $\beta$-Lactamses in the $21^{\text {st }}$ century: characterization, epidemiology, and detection of this important resistance threat. ClinMicrobiol Rev.2001;14:933-51.

[17] Rankin GO, Sutherland $\mathrm{CH}$. Nephrotoxicity of aminoglycosides and cephalosporins in combination. Adverse Drug React Acute Poisoning Rev. 1989;8(2):73-88.

[18] Jeganathan LP, Prakash L, Sivakumar N, Antony A, Alqarawi S, Prajna $L$, et al. Draft genome sequence of an invasive multidrugresistant strain, pseudomonas aeruginosa bk1, isolated from a keratitis patient. Genome Announc. 2014;27(2);2.

[19] Hemavathi, Sarmah P, Shenoy P. Profile of microbial isolates in ophthalmic infections and antibiotic susceptibility of the bacterial isolates: a study in an eye care hospital, bangalore. J Clin Diagn Res. 2014;8(1):23-25.

\section{AUTHOR(S):}

1. Palanisamy Manikandan

\section{PARTICULARS OF CONTRIBUTORS:}

1. Scientist and Head, Department of Microbiology and Molecular Biology, Aravind Eye Hospital and Postgraduate Institute of Ophthalmology, Coimbatore, India.

Assistant Professor, Department of Medical Laboratory Sciences, College of Applied Medical Sciences, Majmaah University, Al-Majmaah, KSA.

\section{NAME, ADDRESS, E-MAIL ID OF THE CORRESPONDING AUTHOR:}

Dr. Palanisamy Manikandan,

Department of Microbiology and Molecular Biology, Aravind Eye Hospital and Postgraduate Institute of Ophthalmology, Avinashi road, Coimbatore-641014, Tamilnadu, India. E-mail: manikandanpalanisamy@gmail.com

FINANCIAL OR OTHER COMPETING INTERESTS: None. 